\title{
A RIGOROUS TREATMENT OF A FOLLOW-THE-LEADER TRAFFIC MODEL WITH TRAFFIC LIGHTS PRESENT*
}

\author{
BRENNA ARGALL ${ }^{\dagger}$, EUGENE CHELESHKIN ${ }^{\dagger}$, J. M. GREENBERG ${ }^{\dagger}$, COLIN HINDE $^{\dagger}$, \\ AND PEI-JEN LIN ${ }^{\dagger}$
}

\begin{abstract}
Traffic flow on a unidirectional roadway in the presence of traffic lights is modeled. Individual car responses to green, yellow, and red lights are postulated and these result in rules governing the acceleration and deceleration of individual cars. The essence of the model is that only specific cars are directly affected by the lights. The other cars behave according to simple followthe-leader rules which limit their speed by the spacing between them and the car directly ahead. The model has a number of desirable properties; namely, cars do not run red lights, cars do not smash into one another, and cars exhibit no velocity reversals. In a situation with multiple lights operating in-phase, we get, after an initial start-up period, a constant number of cars through each light during any green-yellow period. Moreover, this flux is less by one or two cars per period than the flux obtained in discretized versions of the idealized Lighthill-Whitham-Richards model which allows for infinite accelerations.
\end{abstract}

Key words. traffic flow, follow-the-leader, relaxation models, conservation laws

AMS subject classification. 35

PII. S0036139901391215

1. Introduction, model description, and statement of results. In this note we examine the behavior of traffic on a unidirectional highway when multiple traffic lights are present. For simplicity we assume the lights operate in-phase.

The model postulates the dynamics of individual cars but may also be thought of as a coarse discretization of a continuum model introduced recently by Greenberg [1], Aw and Rascle [2], Aw, Klar, Materne, and Rascle [3], and Zhang [9] (details of this correspondence may be found in section $4,(4.6)-(4.8))$.

We assume we are presented with an empirically determined function $s \rightarrow \mathcal{V}(s)$ on $L \leq s$ which satisfies

$$
\begin{gathered}
\mathcal{V}\left(L^{+}\right)=0 \\
\frac{d \mathcal{V}}{d s}(s)>0 \text { and } \frac{d^{2} \mathcal{V}}{d s^{2}}(s)<0, \quad L \leq s<\infty,
\end{gathered}
$$

and

$$
\lim _{s \rightarrow \infty}\left(\mathcal{V}(s), \frac{d \mathcal{V}}{d s}(s), \frac{d^{2} \mathcal{V}}{d s^{2}}(s)\right)=\left(\mathcal{V}_{\infty}>0,0,0\right)
$$

The independent variable $s$ is interpreted as the spacing between cars, $L$ is the minimum car spacing (a lower bound for $L$ is the length of typical car), and $\mathcal{V}_{\infty}>0$

* Received by the editors June 22, 2001; accepted for publication (in revised form) March 1, 2002; published electronically August 28, 2002.

http://www.siam.org/journals/siap/63-1/39121.html

${ }^{\dagger}$ Department of Mathematical Sciences, Carnegie Mellon University, Pittsburgh, PA 15213 (greenber@andrew.cmu.edu). The research of the third author was partially supported by the Applied Mathematical Sciences Program, U.S. Department of Energy and by the U.S. National Science Foundation. 
is the maximum allowable speed of a car. A typical function, and one we shall use in simulations, is

$$
\mathcal{V}(s)=\mathcal{V}_{\infty}\left(1-\frac{L}{s}\right), \quad L \leq s<\infty .
$$

In this classic Lighthill-Whitham-Richards model $[4,5,6,7]$ the function $\mathcal{V}(\cdot)$ gives the velocity of individual cars; in ours it provides an upper bound for the velocity of an individual car. An extensive discussion of suitable functions $\mathcal{V}(\cdot)$ may be found in $[8$, Chapter 4] and the references contained therein. Suffice it to say that the functions $\mathcal{V}(\cdot)$ in our model are consistent with those used in practice.

In this model $x_{k}(t), 1 \leq k \leq N$, denotes the position of the $k$ th car at time $t$, and $0 \leq u_{k}(t)$ is the velocity of the $k$ th car. Throughout,

$$
\frac{d x_{k}}{d t}=u_{k}, \quad 1 \leq k \leq N,
$$

and the cars are ordered so that $\left(x_{k+1}-x_{k}\right)(t) \geq L, 1 \leq k \leq N-1$. During time intervals where the lights are green we assume that

$$
u_{k}=\mathcal{V}\left(\left(x_{k+1}-x_{k}\right)(t)\right)+\alpha_{k}, \quad 1 \leq k \leq N,{ }^{1}
$$

where $\alpha_{k}(t) \leq 0$ satisfies

$$
\epsilon \frac{d \alpha_{k}}{d t}=-\alpha_{k}, \quad 1 \leq k \leq N
$$

The parameter $\epsilon>0$ may be thought of as a relaxation time. Equations (1.6) and (1.7) imply that during the green light periods the velocities, $u_{k}$, satisfy

$$
\frac{d u_{k}}{d t}=\mathcal{V}^{\prime}\left(x_{k+1}-x_{k}\right)\left(u_{k+1}-u_{k}\right)+\frac{\left(\mathcal{V}\left(x_{k+1}-x_{k}\right)-u_{k}\right)}{\epsilon}, \quad 1 \leq k \leq N-1,
$$

and

$$
\frac{d u_{N}}{d t}=\frac{\left(\mathcal{V}_{\infty}-u_{N}\right)}{\epsilon} .
$$

The interesting feature of our model is how yellow or red lights effect the dynamics of an individual car. Our traffic lights cycle from green to yellow to red, and the numbers $0<T G, 0<T Y$, and $0<T R$ denote the duration of the green, yellow, and red lights. At time $t=0$ we assume we have a sequence of $N$ cars located at

$$
x_{k}(0)=\left(k-k_{0}\right) L_{1}, \quad 1 \leq k \leq N,
$$

where $L_{1} \geq L$ (again $L$ is the minimum allowable auto spacing), and we assume these cars are all at rest; i.e.,

$$
u_{k}(0)=0, \quad 1 \leq k \leq N .
$$

Finally, we assume they are at traffic lights located at $x=l_{I}, 1 \leq I \leq M$, where

$$
\left(N-k_{0}\right) L_{1}<l_{1}<l_{2}<\cdots<l_{M} .
$$

\footnotetext{
${ }^{1}$ When $k=N, u_{N}=\mathcal{V}_{\infty}+\alpha_{N}$.
} 
We further assume that each intersection is of width $w>0$ and let

$$
t_{m}=(m-1)(T G+T Y+T R), \quad m=1,2, \ldots,
$$

denote the start of the $m$ th light cycle.

During the time interval $t_{m} \leq t \leq t_{m}+T G$ all cars satisfy (1.5)-(1.7). At time $t_{y} \stackrel{\text { def }}{=} t_{m}+T G$, the green lights turn yellow, and this will have an effect on the traffic flow.

We start by describing what happens to the lead car, the one indexed by $N$, when it encounters a light at $x=l$. We assume that

$$
x_{N}\left(t_{y}\right)<l \text {. }
$$

If

$$
x_{N}\left(t_{y}\right)+u_{N}\left(t_{y}\right) T Y \geq l+w+L,
$$

then the lead car will be able to completely clear the intersection if it travels at its current speed $u_{N}\left(t_{y}\right)$. We allow it to clear the intersection by following its standard dynamics; that is, over the time interval $t_{y} \leq t \leq t_{m+1}$ the $N$ th car satisfies

$$
\frac{d x_{N}}{d t}=u_{N}
$$

where

$$
u_{N}=\mathcal{V}_{\infty}+\alpha_{N}
$$

and $\alpha_{N} \leq 0$ satisfies

$$
\epsilon \frac{d \alpha_{N}}{d t}=-\alpha_{N}
$$

Following these dynamics the lead car accelerates through the intersection.

On the other hand, if

$$
x_{N}\left(t_{y}\right)+u_{N}\left(t_{y}\right) T Y<l+w+L,
$$

then it will be impossible for the $N$ th car to clear the intersection during the yellow phase if it continues to travel at its current speed. If

$$
x_{N}\left(t_{y}\right)+u_{N}\left(t_{y}\right)(T Y+T R) \leq l,
$$

then over the time interval $t_{y} \leq t \leq t_{m+1}$ we require it satisfies the modified dynamics

$$
\frac{d x_{N}}{d t}=u_{N} \text { and } \frac{d u_{N}}{d t}=0
$$

i.e., we insist that it travels at its current speed. This strategy avoids the $N$ th car accelerating and then possibly having to decelerate as it nears the light.

If (1.17) holds and (1.18) is violated, the lead car will have to slow down and possibly stop. When it satisfies the additional inequality

$$
x_{N}\left(t_{y}\right)+u_{N}\left(t_{y}\right)(T Y+T R) / 2>l,
$$


the lead car is mandated to satisfy

$$
\frac{d x_{N}}{d t}=u_{N} \text { and } \frac{d u_{N}}{d t}=\left\{\begin{array}{l}
\frac{-u_{N}^{2}\left(t_{y}\right)}{2\left(l-x_{N}\left(t_{y}\right)\right)}, t_{y} \leq t \leq t_{y}+\frac{2\left(l-x_{N}\left(t_{y}\right)\right)}{u_{N}\left(t_{y}\right)} \\
0, t_{y}+\frac{2\left(l-x_{N}\left(t_{y}\right)\right)}{u_{N}\left(t_{y}\right)} \leq t \leq t_{m+1} \cdot{ }^{2}
\end{array}\right.
$$

This constant deceleration strategy brings the $N$ th car to rest at $x=l$ at $t=t_{y}+$ $\frac{2\left(l-x_{N}\left(t_{y}\right)\right)}{u_{N}\left(t_{y}\right)} \leq t_{m+1}$, and it then sits at the light until $t=t_{m+1}$.

Finally, when

$$
x_{N}\left(t_{y}\right)+u_{N}\left(t_{y}\right)(T Y+T R)>l \text { and } x_{N}\left(t_{y}\right)+u_{N}\left(t_{y}\right)(T Y+T R) / 2 \leq l,
$$

the lead car is mandated to satisfy

$$
\frac{d x_{N}}{d t}=u_{N}(t) \text { and } \frac{d u_{N}}{d t}=\frac{-2\left(x_{N}\left(t_{y}\right)+u_{N}\left(t_{y}\right)(T Y+T G)-l\right)}{(T Y+T G)^{2}}
$$

over the whole interval $t_{y} \leq t \leq t_{m+1}$. This strategy brings the car to the light at $x=l$ at $t_{m+1}$ with velocity

$$
u_{N}\left(t_{m+1}\right)=\frac{2\left(l-x_{N}\left(t_{y}\right)\right)}{(T Y+T R)}-u_{N}\left(t_{y}\right)>0
$$

We note that if the lead car satisfies (1.17), then the cars with indices $k \leq N-1$ follow their standard dynamics $(1.5)-(1.7)$ over $\left[t_{y}, t_{m+1}\right]$ unless they happen to be influenced by some other light at $x=l^{\prime}<l$.

Having described what happens when the lead car encounters a yellow light at $x=l$, we turn our attention to what happens when other cars encounter the same light. We let $k_{l} \leq N-1$ be the largest integer so that

$$
x_{k_{l}}\left(t_{y}\right)<l,
$$

and we let $p_{l} \leq k_{l}$ be the largest integer so that

$$
x_{p_{l}}\left(t_{y}\right)+\min _{p_{l} \leq j \leq k_{l}} u_{j}\left(t_{y}\right) T Y<l+w+L .
$$

The $p_{l}$ th car will be the first one that does not get through the light at $x=l$.

We first consider the situation when $p_{l}<k_{l}$. We assume the existence of a number $\lambda \geq 1$ such that cars travelling with the maximum speed $\mathcal{V}_{\infty}$ can safely brake at a constant deceleration rate $a=\frac{-\mathcal{V}_{\infty}^{2}}{2 \lambda L}$ over a road segment of length $\lambda L$.

We first focus our attention on the situation in which

$$
x_{p_{l}}\left(t_{y}\right)<l-\lambda L .
$$

${ }^{2}$ The dynamics described by (1.21) are equivalent to

$$
\frac{d x_{N}}{d t}=\frac{u_{N}\left(t_{y}\right)\left(l-x_{N}(t)\right)^{1 / 2}}{2\left(l-x_{N}\left(t_{y}\right)\right)^{1 / 2}}, t_{y} \leq t \leq t_{y}+\frac{2\left(l-x_{N}\left(t_{y}\right)\right)}{u_{N}\left(t_{y}\right)}
$$

and

$$
\frac{d x_{N}}{d t}=0, t_{y}+\frac{2\left(l-x_{N}\left(t_{y}\right)\right)}{u_{N}\left(t_{y}\right)} \leq t \leq t_{m+1}
$$


Our basic strategy is to let cars with indices $k \geq p_{l}+1$ follow their standard dynamics (1.5)-(1.7) over $t_{y} \leq t \leq t_{m+1}$. The cars with indices $p_{l}+1 \leq k \leq k_{l}$ will clear the intersection by $t_{m}+T G+T Y \stackrel{\text { def }}{=} t_{r}$; i.e., they will satisfy $x_{k}\left(t_{r}\right) \geq l+w+$ $L$. This follows from the observation that local spatial minima in the velocity are nondecreasing in $t$ (for details see (2.79)-(2.81)).

Rules for the $\boldsymbol{p}_{\boldsymbol{l}}$ th car. So long as $t_{y} \leq t \leq t_{r}$ and $x_{p_{l}}(t)<l-\lambda L$ we let the $p_{l}$ th car follow its standard dynamics (1.5)-(1.7). If there is a first $t_{p_{l}}<t_{r}$ so that $x_{p_{l}}\left(t_{p_{l}}\right)=l-\lambda L$, then the driver must decide what to do. In the unlikely event that

$$
u_{p_{l}}\left(t_{p_{l}}\right)\left(t_{m+1}-t_{p_{l}}\right) \leq \lambda L,
$$

then over the interval $\left[t_{p_{l}}, t_{m+1}\right]$ the $p_{l}$ th car is required to satisfy

$$
\frac{d x_{p_{l}}}{d t}=\min \left(u_{p_{l}}\left(t_{y}\right), U_{p_{l}}(t)\right) \stackrel{\text { def }}{=} u_{p_{l}}(t)
$$

and

$$
\begin{array}{r}
\frac{d U_{p_{l}}}{d t}=\mathcal{V}^{\prime}\left(x_{p_{l}+1}-x_{p_{l}}\right)\left(u_{p_{l}+1}-U_{p_{l}}\right) \\
+\frac{\left(\mathcal{V}\left(x_{p_{l}+1}-x_{p_{l}}\right)-U_{p_{l}}\right)}{\epsilon}
\end{array}
$$

and

$$
U_{p_{l}}\left(t_{y}\right)=u_{p_{l}}\left(t_{y}\right)
$$

On the other hand, if

$$
u_{p_{l}}\left(t_{p_{l}}\right)\left(t_{m+1}-t_{p_{l}}\right)>\lambda L,
$$

then the $p_{l}$ th car will have to slow down and possibly stop.

When the $p_{l}$ th car satisfies the additional inequality

$$
u_{p_{l}}\left(t_{p_{l}}\right)\left(t_{m+1}-t_{p_{l}}\right) / 2>\lambda L,
$$

the $p_{l}$ th car is required to satisfy

$$
\frac{d x_{p_{l}}}{d t}=\min \left(\frac{u_{p_{l}}\left(t_{p_{l}}\right)\left(l-x_{p_{l}}\right)^{1 / 2}}{2(\lambda L)^{1 / 2}}, U_{p_{l}}\right) \stackrel{d e f}{=} u_{p_{l}},
$$

where

$$
\frac{d U_{p_{l}}}{d t}=\mathcal{V}^{\prime}\left(x_{p_{l}+1}-x_{p_{l}}\right)\left(u_{p_{l}+1}-U_{p_{l}}\right)+\frac{\left(\mathcal{V}\left(x_{p_{l}+1}-x_{p_{l}}\right)-U_{p_{l}}\right)}{\epsilon}
$$

and

$$
x_{p_{l}}\left(t_{p_{l}}\right)=l-\lambda L \text { and } U_{p_{l}}\left(t_{p_{l}}\right)=u_{p_{l}}\left(t_{p_{l}}\right) .
$$

When (1.31) reduces to

$$
\frac{d x_{p_{l}}}{d t}=\frac{u_{p_{l}}\left(t_{p_{l}}\right)\left(l-x_{p_{l}}\right)^{1 / 2}}{2(\lambda L)^{1 / 2}} \stackrel{\text { def }}{=} v_{p_{l}}
$$


we see that

$$
\frac{d v_{p_{l}}}{d t}=-\frac{u_{p_{l}}^{2}\left(t_{p_{l}}\right)}{2 \lambda L} \leq-\frac{\mathcal{V}_{\infty}^{2}}{2 \lambda L}
$$

and thus we apply this constant braking strategy over $t_{p_{l}} \leq t \leq t_{p_{l}}+\frac{2 \lambda L}{u_{p_{l}}\left(t_{p_{l}}\right)}$ and the strategy $x_{p_{l}}(t)=l$ over $t_{p_{l}}+\frac{2 \lambda L}{u_{p_{l}}\left(t_{p_{l}}\right)} \leq t \leq t_{m+1}$.

If instead of (1.30) the $p_{l}$ th car satisfies

$$
u_{p_{l}}\left(t_{p_{l}}\right)\left(t_{m+1}-t_{p_{l}}\right) / 2 \leq \lambda L,
$$

the $p_{l}$ th car is required to satisfy

$$
\begin{aligned}
& \frac{d x_{p_{l}}}{d t}=\min \left(u_{p_{l}}\left(t_{p_{l}}\right)+\frac{2 \lambda L-u_{p_{l}}\left(t_{p_{l}}\right)\left(t_{m+1}-t_{p_{l}}\right)}{\left(t_{m+1}-t_{p_{l}}\right)^{2}}\left(t-t_{p_{l}}\right), U_{p_{l}}\right) \\
& \quad \stackrel{\text { def }}{=} u_{p_{l}}, \quad t_{p_{l}} \leq t \leq t_{m+1}
\end{aligned}
$$

and (1.33), and again $U_{p_{l}}$ satisfies (1.32) and $(1.33)_{2}$.

The dynamics for $U_{p_{l}}$ postulated in (1.28) and (1.32) might seem a bit strange. What we are insisting is that the $p_{l}$ th car must travel no faster than the minimum of its braking speed and the speed that it would travel at if it disregarded the light and allowed its velocity to be determined by the car ahead. The latter speed $U_{p_{l}}$ is computed from the standard dynamics equation (see (1.6), (1.7), (1.7a), and (1.7b)).

If there is no such time $t_{p_{l}}<t_{r}$ so that $x_{p_{l}}\left(t_{p_{l}}\right)=l-\lambda L$, then we know that $x_{p_{l}}\left(t_{r}\right) \leq l-\lambda L$. In this situation we replace $t_{p_{l}}$ in (1.27)-(1.37) by $t_{r}$ and the terms $\lambda L$ in all inequalities and identities by $l-x_{p_{l}}\left(t_{r}\right)$.

Finally, if (1.26) does not hold, i.e., if

$$
l-\lambda L \leq x_{p_{l}}\left(t_{y}\right)<l,
$$

we set $t_{p_{l}}$ to $t_{y}$ in (1.27)-(1.37) and replace $\lambda L$ in these formulas by $l-x_{p_{l}}\left(t_{y}\right)$.

The rules when $p_{l}=k_{l}$ are similarly amended.

The cars with indices $p_{l-1} \leq k \leq p_{l}-1$ are required to satisfy their standard dynamics over $\left[t_{y}, t_{m+1}\right]$.

Our first result deals with the model's consistency; we shall show that for all $t \geq 0$ and all indices, $L \leq\left(x_{k+1}-x_{k}\right)(t)$ and $0 \leq u_{k}(t)<\mathcal{V}\left(\left(x_{k+1}-x_{k}\right)(t)\right)$. We also have the theorem that no cars run any red lights. With two in-phase lights, the number of cars through an intersection during the green and yellow phases is, after a start-up period, a constant. This constant is less than the constant obtained with models which allow for infinite accelerations, i.e., discrete Lagrangian versions of the Lighthill-Whitham-Richards model $[4,5,6,7]$.

One surprising observation about the model just described is that the largest decelerations are not necessarily associated with the cars indexed by $p_{l}$ but rather cars with indices $k \leq p_{l}-1$ which are forced to slow down because the $p_{l}$ th car has stopped. Equation (1.7a) implies that the latter cars' decelerations are determined by the negative velocity gradients $u_{k+1}-u_{k}$.

Finally, we note that though we have been quite specific in postulating our stopping rules for the $p_{l}$ th car, it would have sufficed to have chosen any rule of the form

$$
\frac{d x_{p_{l}}}{d t}=\min \left(v_{p_{l}}, U_{p_{l}}\right) \stackrel{\text { def }}{=} u_{p_{l}}, \quad t_{p_{l}} \leq t \leq t_{m+1},
$$


where $U_{p_{l}}$ satisfies

$$
\frac{d U_{p_{l}}}{d t}=\mathcal{V}^{\prime}\left(x_{p_{l+1}}-x_{p_{l}}\right)\left(u_{p_{l+1}}-U_{p_{l}}\right)+\left(\mathcal{V}\left(x_{p_{l+1}}-x_{p_{l}}\right)-U_{p_{l}}\right) \epsilon
$$

and $U_{p_{l}}\left(t_{y}\right)=u_{p_{l}}\left(t_{y}\right)$ if $p_{l} \leq N-1$, and

$$
\frac{d U_{N}}{d t}=\frac{\left(\mathcal{V}_{\infty}-U_{N}\right)}{\epsilon} \text { and } U_{N}\left(t_{y}\right)=u_{p_{l}}\left(t_{y}\right)
$$

if $p_{l}=N$, and where $v_{p_{l}} \geq 0$ is chosen so that if

$$
\frac{d x_{p_{l}}}{d t}=v_{p_{l}}, \quad t_{y} \leq t \leq t_{m+1} \text { and } x_{p_{l}}\left(t_{y}\right)<l,
$$

then $x_{p_{l}}(t) \leq l, t_{y} \leq t \leq t_{m+1}$.

2. Model consistency. In this section we turn our attention to the issue of model consistency. The central issue before us is to show that for $1 \leq k \leq N-1$ and $0 \leq t$

$$
L \leq\left(x_{k+1}-x_{k}\right)(t) \text { and } 0 \leq u_{k}(t)<\mathcal{V}\left(\left(x_{k+1}-x_{k}\right)(t)\right)
$$

and that for $k=N$ and $0 \leq t$

$$
0 \leq u_{N}(t) \leq \mathcal{V}_{\infty}
$$

We are also interested in knowing that the distinguished cars indexed by $p_{l}$ do not run the red lights over the intervals $t_{r} \stackrel{\text { def }}{=}(m-1)(T G+T Y+T R)+T G+T Y \leq$ $t \leq m(T G+T Y+T R) \stackrel{\text { def }}{=} t_{m+1}$ and that the $\left(p_{l}+1\right)$ st car clears the intersection by $t_{r}$, i.e., satisfies

$$
x_{p_{l}+1}\left(t_{r}\right) \geq l+w+L .
$$

Once again $x=l$ is supposed to be the leading edge of the intersection, $w$ the width of the intersection, and $L$ the length of a car.

There are two natural approaches that one can take to establish the above claims. The first is to show that the desired conclusions follow directly from the governing differential equations and initial and constraining conditions while the second is to show that approximate solutions, generated by numerical discretization, satisfy the desired consistency results. Noting then that these consistency results are sufficient to guarantee that the approximate solutions converge (as $\Delta t \rightarrow 0$ ) to solutions of the original model, we are guaranteed that these limiting solutions satisfy the same consistency results. We adopt the latter procedure here since in the next section we shall perform computations with the discrete approximating system.

Throughout, $\Delta t$ will denote our time step and the quantities $\left(x_{k}^{n}, u_{k}^{n}, \alpha_{k}^{n}\right)$ will denote the values of the approximate solutions at $t_{n}=n \Delta t$. To keep matters simple we shall assume that the numbers $T G / \Delta t, T Y / \Delta t, T R / \Delta t$, and $\epsilon / \Delta t$ are all integers and we shall assume that $\Delta t \leq \min \left(\epsilon,\left(\mathcal{V}^{\prime}(L)=\max _{L \leq s} \mathcal{V}^{\prime}(s)\right)^{-1}\right)$.

Our first result deals with the traffic flow over the time intervals

$$
t_{m} \stackrel{\text { def }}{=}(m-1)(T G+T Y+T R) \leq t_{n}=n \Delta t \leq t_{y} \stackrel{\text { def }}{=} t_{m}+T G
$$


when all lights are green. Over such intervals we replace (1.5) by

$$
x_{k}^{n+1}=x_{k}^{n}+u_{k}^{n} \Delta t, \quad 1 \leq k \leq N,
$$

and this yields

$$
s_{k}^{n+1}=s_{k}^{n}+\left(u_{k+1}^{n}-u_{k}^{n}\right) \Delta t, \quad 1 \leq k \leq N-1,
$$

where

$$
s_{k}^{n}=\left(x_{k+1}^{n}-x_{k}^{n}\right) \text { and } s_{k}^{n+1}=\left(x_{k+1}^{n+1}-x_{k}^{n+1}\right) .
$$

The $u$ 's and $s$ 's are related by

$$
u_{k}^{n}=\mathcal{V}\left(s_{k}^{n}\right)+\alpha_{k}^{n}
$$

and

$$
u_{k}^{n+1}=\mathcal{V}\left(s_{k}^{n+1}\right)+\left(1-\frac{\Delta t}{\epsilon}\right) \alpha_{k}^{n} .
$$

These updates hold for indices $n$ satisfying

$$
(m-1)(T G+T Y+T R) / \Delta t \stackrel{\text { def }}{=} n_{m} \leq n \leq n_{m}+T G / \Delta t-1 .
$$

Theorem 1. Suppose that

$$
L \leq s_{k}^{n_{m}} \quad \text { and } \quad 0 \leq u_{k}^{n_{m}} \leq \mathcal{V}\left(s_{k}^{n_{m}}\right), \quad 1 \leq k \leq N-1,
$$

and

$$
0 \leq u_{N}^{n_{m}} \leq \mathcal{V}_{\infty}=\lim _{s \rightarrow \infty} \mathcal{V}(s)
$$

Then, the same inequalities hold for

$$
n_{m} \leq n \leq n_{m}+T G / \Delta t \stackrel{\text { def }}{=} n_{y} .
$$

Proof. The identity (2.6) implies that if $s_{k}^{n} \geq L$ and $u_{k+1}^{n}-u_{k}^{n} \geq 0$, then $s_{k}^{n+1} \geq$ $s_{k}^{n} \geq L$. In the situation in which $u_{k+1}^{n}-u_{k}^{n}<0,(2.6)$ implies that

$$
s_{k}^{n+1}=s_{k}^{n}+\left(u_{k+1}^{n}-\alpha_{k}^{n}-\mathcal{V}\left(s_{k}^{n}\right)\right) \Delta t
$$

and the natural induction hypotheses $\alpha_{k}^{n} \leq 0,0 \leq u_{k}^{n} \leq \mathcal{V}\left(s_{k}^{n}\right)$, and $s_{k}^{n} \geq L$ imply that $u_{k+1}^{n}-\alpha_{k}^{n} \geq 0$. In the situation in which $0 \leq u_{k+1}^{n}-\alpha_{k}^{n}<\mathcal{V}_{\infty}$ we are guaranteed a unique $\bar{s}_{k+1}^{n} \in[L, \infty)$ satisfying

$$
u_{k+1}^{n}-\alpha_{k}^{n}=\mathcal{V}\left(\bar{s}_{k+1}^{n}\right),
$$

and here (2.14) reduces to

$$
s_{k}^{n+1}=s_{k}^{n}+\left(\mathcal{V}\left(\bar{s}_{k+1}^{n}\right)-\mathcal{V}\left(s_{k}^{n}\right)\right) \Delta t
$$

or

$$
s_{k}^{n+1}=\left(1-\mathcal{V}^{\prime}\left(s_{*}\right) \Delta t\right) s_{k}^{n}+\mathcal{V}^{\prime}\left(s_{*}\right) \Delta t \bar{s}_{k}^{n}
$$


for some $s_{*} \in\left(\min \left(s_{k}^{n}, \bar{s}_{k+1}^{n}\right), \max \left(s_{k}^{n}, \bar{s}_{k+1}^{n}\right)\right)$. The latter identity, together with

$$
\Delta t \mathcal{V}^{\prime}(L) \leq 1 \text { and } \min \left(s_{k}^{n}, \bar{s}_{k+1}^{n}\right) \geq L,
$$

yields $s_{k}^{n+1} \geq L$. When $u_{k+1}^{n}-u_{k}^{n}<0$ and $u_{k+1}^{n}-\alpha_{k}^{n} \geq \mathcal{V}_{\infty}$, the identity (2.14) implies that

$$
s_{k}^{n+1} \geq s_{k}^{n}+\left(\mathcal{V}_{\infty}-\mathcal{V}\left(s_{k}^{n}\right)\right) \Delta t
$$

The inequality $(2.18)_{1}$ guarantees that $s \rightarrow s+\left(\mathcal{V}_{\infty}-\mathcal{V}(s)\right) \Delta t$ is strictly increasing on $[L, \infty)$ and thus (2.19) implies that $s_{k}^{n+1} \geq L+\mathcal{V}_{\infty} \Delta t \geq L$ as desired.

The induction hypothesis $\alpha_{k}^{n} \leq 0$ together with $\Delta t / \epsilon \leq 1$ and (2.9) guarantees that $u_{k}^{n+1} \leq \mathcal{V}\left(s_{k}^{n+1}\right)$. What remains to be shown is that $u_{k}^{n+1} \geq 0$. To establish this assertion we combine (2.8) and (2.9) to obtain

$$
u_{k}^{n+1}=\mathcal{V}\left(s_{k}^{n}+\left(u_{k+1}^{n}-u_{k}^{n}\right) \Delta t\right)+\left(1-\frac{\Delta t}{\epsilon}\right)\left(u_{k}^{n}-\mathcal{V}\left(s_{k}^{n}\right)\right)
$$

Noting that

$$
\mathcal{V}\left(s_{k}^{n}+\left(u_{k+1}^{n}-u_{k}^{n}\right) \Delta t\right)=\mathcal{V}\left(s_{k}^{n}\right)+\mathcal{V}^{\prime}\left(s_{\#}\right)\left(u_{k+1}^{n}-u_{k}^{n}\right) \Delta t
$$

for some $s_{\#} \geq L$, we find that

$$
u_{k}^{n+1}=\mathcal{V}^{\prime}\left(s_{\#}\right) \Delta t u_{k+1}^{n}+\frac{\Delta t}{\epsilon}\left(\mathcal{V}\left(s_{k}^{n}\right)-u_{k}^{n}\right)+\left(1-\mathcal{V}^{\prime}\left(s_{\#}\right) \Delta t\right) u_{k}^{n} .
$$

The last identity, when combined with

$$
\Delta t \mathcal{V}^{\prime}\left(s_{\#}\right) \leq 1, \quad \Delta t / \epsilon \leq 1, \quad u_{k}^{n} \geq 0, \quad u_{k+1}^{n} \geq 0, \quad \text { and } \quad \mathcal{V}\left(s_{k}^{n}\right)-u_{k}^{n} \geq 0,
$$

yields $u_{k}^{n+1} \geq \min \left(u_{k}^{n}, u_{k+1}^{n}\right) \geq 0$ as desired.

We now turn our attention to what happens over the yellow and red phases, i.e., when

$$
t_{y} \stackrel{\text { def }}{=}(m-1)(T G+T Y+T R)+T G \leq t_{n}=n \Delta t<t_{m+1} \stackrel{\text { def }}{=} m(T G+T Y+T R) .
$$

The results of Theorem 1 imply that when $n=n_{y} \stackrel{\text { def }}{=}(m-1)(T G+T Y+T R)+T G / \Delta t$ the following inequalities are valid:

$$
L \leq s_{k}^{n_{y}} \text { and } 0<u_{k}^{n_{y}} \leq \mathcal{V}\left(s_{k}^{n_{y}}\right), \quad 1 \leq k \leq N-1,
$$

and

$$
0 \leq u_{N}^{n_{y}} \leq \mathcal{V}_{\infty}=\lim _{s \rightarrow \infty} \mathcal{V}(s)
$$

Our next goal is to show that (2.21) and (2.22) hold for indices

$$
n_{y} \leq n \leq n_{m+1} \stackrel{\text { def }}{=} m(T G+T Y+T R) .
$$

For definiteness we assume the lights are located at $l_{1}<l_{2}<\cdots<l_{M}$ where $M<<N$ and that $L<<l_{I+1}-l_{I}, 1 \leq I \leq M-1$. For $1 \leq I \leq M, k_{I}$ will be the largest integer less than or equal to $N$, so that

$$
x_{k_{I}}^{n_{y}}<l_{I}
$$


and $p_{I}$ will be the largest integer less than or equal to $k_{I}$ so that

$$
x_{p_{I}}^{n_{y}}+\left(\min _{p_{I} \leq j \leq k_{I}} u_{j}^{n_{y}}\right) T Y<l_{I}+w+L .
$$

It can and does happen that for some $I<M$

$$
p_{I}=p_{I+1}=\cdots=p_{M}=N .
$$

Our first task is to establish the desired inequalities for indices $\left(p_{I-1}+1\right) \leq k \leq$ $p_{I}=N$ for $n_{y} \leq n \leq n_{m+1}$. This is the situation that is obtained when the lead car, indexed by $N$, has passed the $(I-1)$ st light but not the $I$ th light.

The rules laid out in (1.17)-(1.23) imply that $x_{N}(\cdot)$ satisfies

$$
\frac{d x_{N}}{d t}=\min \left(v_{N}, U_{N}\right) \stackrel{\text { def }}{=} u_{N}, \quad t_{y} \leq t \leq t_{m+1},
$$

where $U_{N}$ satisfies

$$
\frac{d U_{N}}{d t}=\frac{\left(\mathcal{V}_{\infty}-U_{N}\right)}{\epsilon} \text { and } U_{N}\left(t_{y}\right)=u_{N}\left(t_{y}\right),
$$

and $v_{N}(\cdot) \geq 0$ is chosen so that if $x_{N}(\cdot)$ satisfies

$$
\frac{d x_{N}}{d t}=v_{N} \text { and } x_{N}\left(t_{y}\right)<l_{I},
$$

then $x_{N}\left(t_{m+1}\right) \leq l_{I}$. We replace this system with its discrete analogue,

$$
\begin{gathered}
x_{N}^{n+1}=x_{N}^{n}+u_{N}^{n} \Delta t, \quad n_{y} \leq n \leq n_{m+1}-1, \\
U_{N}^{n+1}=\mathcal{V}_{\infty}+\left(1-\frac{\Delta t}{\epsilon}\right)\left(U_{N}^{n}-\mathcal{V}_{\infty}\right), \quad n_{y} \leq n \leq n_{m+1}-1,
\end{gathered}
$$

and these are solved subject to the initial conditions

$$
x_{N}^{n_{y}}<l_{I} \text { and } 0 \leq u_{N}^{n_{y}} \leq U_{N}^{n_{y}} \leq \mathcal{V}_{\infty} .
$$

The discrete velocity $u_{N}^{n}$ is given by

$$
u_{N}^{n}=\min \left(v_{N}^{n}, U_{N}^{n}\right),
$$

and $v_{N}^{n} \geq 0$ is a discretization of $v_{N}$ with the property that if

$$
x_{N}^{n+1}=x_{N}^{n}+v_{N}^{n} \Delta t \text { and } x_{N}^{n_{y}}<l_{I}
$$

for $n_{y} \leq n \leq n_{m+1}-1$, then

$$
x_{N}^{n_{m+1}} \leq l_{I} .
$$

The identities (2.31), $(2.32)_{2}$, and (2.33) guarantee that

$$
0 \leq u_{N}^{n} \leq \mathcal{V}_{\infty}, \quad n_{y} \leq n \leq n_{m+1} .
$$


If we assume that $\left(p_{I-1}+1\right) \leq N-1$, then the $(N-1)$ st car will follow the standard dynamics (1.5)-(1.7) on $t_{y} \leq t \leq t_{m+1}$, and thus for $n_{y} \leq n \leq n_{m+1}-1$ we have the approximating discrete system:

$$
\begin{aligned}
x_{N-1}^{n+1} & =x_{N-1}^{n}+u_{N-1}^{n} \Delta t, \quad u_{N-1}^{n}=\mathcal{V}\left(s_{N-1}^{n}\right)+\alpha_{N-1}^{n}, \\
\text { and } u_{N-1}^{n+1} & =\mathcal{V}\left(s_{N-1}^{n+1}\right)+\left(1-\frac{\Delta t}{\epsilon}\right) \alpha_{N-1}^{n},
\end{aligned}
$$

where

$$
s_{N-1}^{n}=x_{N}^{n}-x_{N-1}^{n} \text { and } s_{N-1}^{n+1}=x_{N}^{n+1}-x_{N-1}^{n+1}=s_{N-1}^{n}+\left(u_{N}^{n}-u_{N-1}^{n}\right) \Delta t .
$$

The inequalities (2.21) and (2.22) imply that $\alpha_{N-1}^{n_{y}} \leq 0, \alpha_{N}^{n_{y}} \leq 0$, and $s_{N-1}^{n_{y}} \geq L$. The identities (2.37) and (2.38) imply that

$$
s_{N-1}^{n+1}=s_{N-1}^{n}+\left(u_{N}^{n}-\left(1-\frac{\Delta t}{\epsilon}\right)^{n} \alpha_{N-1}^{n_{y}}-\mathcal{V}\left(s_{N-1}^{n}\right)\right) \Delta t,
$$

and $(2.37)_{2}$ and (2.39), together with

$$
L \leq s_{N-1}^{n_{y}}, \quad \alpha_{N-1}^{n_{y}} \leq 0, \quad u_{N}^{n} \geq 0, \quad \Delta t \mathcal{V}^{\prime}(L) \leq 1, \quad \text { and } \quad \Delta t \leq \epsilon
$$

and the arguments used to establish Theorem 1, imply that

$$
L \leq s_{N-1}^{n}, \quad n_{y} \leq n \leq n_{m+1} .
$$

The arguments used to establish Theorem 1 along with (2.40) and (2.41) also yield $0 \leq u_{N-1}^{n} \leq \mathcal{V}\left(s_{N-1}^{n}\right), n_{y} \leq n \leq n_{m+1}$. An induction on $k$ for indices $\left(p_{I-1}+1\right) \leq k$ then yields

$$
L \leq s_{k}^{n}=\left(x_{k+1}^{n}-x_{k}^{n}\right) \text { and } 0 \leq u_{k}^{n} \leq \mathcal{V}\left(s_{k}^{n}\right), \quad n_{y} \leq n \leq n_{m+1} .
$$

This situation when $p_{I-1}=N-1$ is handled similarly, provided that one adopts the proper first order integration scheme for $U_{N-1}$. The governing equation for $U_{N-1}$ is

$$
\frac{d U_{N-1}}{d t}=\mathcal{V}^{\prime}\left(x_{N}-x_{N-1}\right)\left(u_{N}-U_{N-1}\right)+\frac{\left(\mathcal{V}\left(x_{N}-x_{N-1}\right)-U_{N-1}\right)}{\epsilon},
$$

where

$$
\frac{d\left(x_{N}-x_{N-1}\right)}{d t}=u_{N}-u_{N-1},
$$

and $v_{N-1} \geq 0$ is chosen so that if

$$
\frac{d x_{N}}{d t}=v_{N-1} \text { and } x_{N-1}\left(t_{y}\right)<l_{I},
$$

then

$$
x_{N-1}\left(t_{m+1}\right) \leq l_{I} .
$$

Additionally

$$
u_{N-1} \stackrel{\text { def }}{=} \min \left(v_{N-1}, U_{N-1}\right) .
$$


The integration scheme we use is

$$
U_{N-1}^{n+1}=\mathcal{V}\left(s_{N-1}^{n}+\left(u_{N}^{n}-U_{N-1}^{n}\right) \Delta t\right)+\left(1-\frac{\Delta t}{\epsilon}\right)\left(U_{N-1}^{n}-\mathcal{V}\left(s_{N-1}^{n}\right)\right),{ }^{3}
$$

where

$$
s_{N-1}^{n}=x_{N}^{n}-x_{N-1}^{n} .
$$

To complete the proof one does an induction on the index $I$, first replacing $I$ by $I-1$. One knows that the car with index $\left(p_{I-1}+1\right)$ has a velocity $u_{\left(p_{I-1}+1\right)}^{n}$ satisfying

$$
0 \leq u_{\left(p_{I-1}+1\right)}^{n} \leq \mathcal{V}\left(s_{p_{I-1}+1}^{n}\right), \quad n_{y} \leq n \leq n_{m+1} .
$$

We first focus on the $p_{I-1}$ st car and note that

$$
\frac{d x_{p_{I-1}}}{d t}=\min \left(v_{p_{I-1}}, U_{p_{I-1}}\right) \stackrel{\text { def }}{=} u_{p_{I-1}},
$$

and

$$
\frac{d s_{p_{I-1}}}{d t}=\left(u_{\left(p_{I-1}+1\right)}-u_{p_{I-1}}\right) .
$$

The rules laid out in (1.7)-(1.23) imply that

$$
\frac{d U_{p_{I-1}}}{d t}=\mathcal{V}^{\prime}\left(s_{\left(p_{I-1}\right)}\right)\left(u_{\left(p_{I-1}+1\right)}-U_{p_{I-1}}\right)+\frac{\left(\mathcal{V}\left(s_{\left(p_{I-1}+1\right)}\right)-U_{p_{I-1}}\right)}{\epsilon}
$$

and that the velocity field $0 \leq v_{p_{I-1}}$ is chosen so that if $x_{p_{I-1}}$ evolves as

$$
\frac{d x_{p_{I-1}}}{d t}=v_{p_{I-1}} \text { and } x_{p_{I-1}}\left(t_{y}\right)<l_{I},
$$

then

$$
x_{p_{I-1}}\left(t_{m+1}\right) \leq l_{I-1} .
$$

The discretization we apply to the $p_{I-1}$ st car is

$$
x_{p_{I-1}}^{n+1}=x_{p_{I-1}}^{n}+u_{p_{I-1}}^{n} \Delta t \text { and } s_{p_{I-1}}^{n+1}=s_{p_{I-1}}^{n}+\left(u_{\left(p_{I-1}+1\right)}^{n}-u_{p_{I-1}}^{n}\right) \Delta t
$$

for $n_{y} \leq n \leq n_{m+1}-1$. Moreover, for some $n_{y} \leq n_{0} \leq n_{y}+T Y / \Delta t-1$

$$
u_{p_{I-1}}^{n+1}=\mathcal{V}\left(s_{p_{I-1}}^{n+1}\right)+\left(1-\frac{\Delta t}{\epsilon}\right)\left(u_{p_{I-1}}^{n}-\mathcal{V}\left(s_{p_{I-1}}^{n}\right)\right)
$$

and

$$
U_{p_{I-1}}^{n+1}=u_{p_{I-1}}^{n+1},
$$

${ }^{3}$ This scheme is essentially a first order Euler scheme applied to (2.43). The scheme implies that

$$
U_{N-1}^{n+1}=U_{N-1}^{n}+\Delta t \mathcal{V}^{\prime}\left(s_{N-1}^{n}\right)\left(u_{N}^{n}-U_{N-1}^{n}\right)+\frac{\Delta t}{\epsilon}\left(\mathcal{V}\left(s_{N-1}^{n}\right)-U_{N-1}^{n}\right)+0(\Delta t)^{2}
$$


whereas for $n_{0} \leq n \leq n_{m+1}-1$

$$
u_{p_{I-1}}^{n}=\min \left(v_{p_{I-1}}^{n}, U_{p_{I-1}}^{n}\right)
$$

$$
U_{p_{I-1}}^{n+1}=\mathcal{V}\left(s_{p_{I-1}}^{n}+\left(u_{\left(p_{I-1}+1\right)}^{n}-U_{p_{I-1}}^{n}\right) \Delta t\right)+\left(1-\frac{\Delta t}{\epsilon}\right)\left(U_{p_{I-1}}^{n}-\mathcal{V}\left(s_{p_{I-1}}^{n}\right)\right),{ }^{4}
$$

and

$$
U_{p_{I-1}}^{n_{0}}=u_{p_{I-1}}^{n_{0}} \text { and } x_{p_{I-1}}^{n_{0}}<l_{I} .
$$

Finally $v_{p_{I-1}}^{n}$ is chosen so that if

$$
x_{p_{I-1}}^{n+1}=x_{p_{I-1}}^{n}+v_{p_{I-1}}^{n} \Delta t, \quad n_{0} \leq n \leq n_{m+1}-1,
$$

then

$$
x_{p_{I-1}}^{n_{m+1}} \leq l_{I} .
$$

The arguments employed to establish Theorem 1 guarantee that for $n_{y} \leq n \leq n_{0}$

$$
L \leq s_{p_{I-1}}^{n} \text { and } 0 \leq u_{p_{I-1}}^{n} \leq \mathcal{V}\left(s_{p_{I-1}}^{n}\right)
$$

and that for $n=n_{0}$

$$
0 \leq u_{p_{I-1}}^{n_{0}} \leq U_{p_{I-1}}^{n_{0}} \leq \mathcal{V}\left(s_{p_{I-1}}^{n_{0}}\right) .
$$

LEMma 1. For $n_{0} \leq n \leq n_{m+1}$

$$
L \leq s_{p_{I-1}}^{n} \text { and } 0 \leq u_{p_{I-1}}^{n} \leq U_{p_{I-1}}^{n} \leq \mathcal{V}\left(s_{p_{I-1}}^{n}\right) .
$$

Proof. The identities (2.56) and (2.60) imply that

$$
\begin{aligned}
& \mathcal{V}\left(s_{p_{I-1}}^{n+1}\right)-U_{p_{I-1}}^{n+1}= \mathcal{V}\left(s_{p_{I-1}}^{n}+\left(u_{\left(p_{I-1}+1\right)}^{n}-u_{p_{I-1}}^{n}\right) \Delta t\right) \\
&-\mathcal{V}\left(s_{p_{I-1}}^{n}+\left(u_{\left(p_{I-1}+1\right)}^{n}-U_{p_{I-1}}^{n}\right) \Delta t\right) \\
&+\left(1-\frac{\Delta t}{\epsilon}\right)\left(\mathcal{V}\left(s_{p_{I-1}}^{n}\right)-U_{p_{I-1}}^{n}\right) \\
&=\Delta t \mathcal{V}^{\prime}\left(s_{\#}\right)\left(U_{p_{I-1}}^{n}-u_{p_{I-1}}^{n}\right)+\left(1-\frac{\Delta t}{\epsilon}\right)\left(\mathcal{V}\left(s_{p_{I-1}}^{n}\right)-U_{p_{I-1}}^{n}\right)
\end{aligned}
$$

for some $s_{\#} \geq \min \left(s_{p_{I-1}}^{n}+\left(u_{\left(p_{I-1}+1\right)}^{n}-u_{p_{I-1}}^{n}\right) \Delta t, s_{p_{I-1}}^{n}+\left(u_{\left(p_{I-1}+1\right)}^{n}-U_{p_{I-1}}^{n}\right) \Delta t\right)$. If we now make the induction hypotheses that

$$
L \leq s_{p_{I-1}}^{n} \text { and } 0 \leq U_{p_{I-1}}^{n} \leq \mathcal{V}\left(s_{p_{I-1}}^{n}\right),
$$

then (2.59) implies that

$$
0 \leq u_{p_{I-1}}^{n} \leq U_{p_{I-1}}^{n} \leq \mathcal{V}\left(s_{p_{I-1}}^{n}\right)
$$

\footnotetext{
${ }^{4}$ See Footnote 3.
} 
and (2.69) and (2.42) with $k=p_{I-1}+1$ implies that

$$
\begin{aligned}
& \min \left(s_{p_{I-1}}^{n}+\left(u_{\left(p_{I-1}+1\right)}^{n}-u_{p_{I-1}}^{n}\right) \Delta t, s_{p_{I-1}}^{n}+\left(u_{\left(p_{I-1}+1\right)}^{n}-U_{p_{I-1}}^{n}\right) \Delta t\right) \\
& \geq s_{p_{I-1}}^{n}-\mathcal{V}\left(s_{p_{I-1}}^{n}\right) \Delta t \stackrel{\text { def }}{=} \mathcal{F}\left(s_{p_{I-1}}^{n}\right) .
\end{aligned}
$$

This constraint $\Delta t \mathcal{V}^{\prime}(s) \leq 1, L \leq s$ guarantees $\mathcal{F}(\cdot)$ in nondecreasing on $L \leq s$, and this fact, together with $\mathcal{F}(L)=L$, guarantees that $s_{p_{I-1}}^{n+1}$ and $s_{\#}$ are both greater than or equal to $L$. Moreover, (2.67) also yields $U_{p_{I-1}}^{n+1} \leq \mathcal{V}\left(s_{p_{I-1}}^{n}\right)$. The defining relation (2.60) and (2.70) and $u_{\left(p_{I-1}+1\right)}^{n} \geq 0$ also implies that

$$
U_{p_{I-1}}^{n+1}=\Delta t \mathcal{V}^{\prime}\left(s_{*}\right) u_{\left(p_{I-1}+1\right)}^{n}+\left(1-\Delta t \mathcal{V}^{\prime}\left(s_{*}\right)\right) U_{p_{I-1}}^{n}+\frac{\Delta t}{\epsilon}\left(\mathcal{V}\left(s_{p_{I-1}}^{n}\right)-U_{p_{I-1}}^{n}\right)
$$

for some $s_{*} \geq L$ and (2.71) guarantees that $U_{p_{I-1}}^{n+1} \geq 0$. The last inequality and (2.59), with $n+1$, guarantees that

$$
0 \leq u_{p_{I-1}}^{n+1} \leq U_{p_{I-1}}^{n+1} \leq \mathcal{V}\left(s_{p_{I-1}}^{n+1}\right),
$$

and this completes the proof of Lemma 1.

Once again an induction on $k$ for indices $\left(p_{I-2}+1\right) \leq k$ yields

$$
L \leq s_{k}^{n}=\left(x_{k+1}^{n}-x_{k}^{n}\right) \text { and } 0 \leq u_{k}^{n} \leq \mathcal{V}\left(s_{k}^{n}\right)
$$

and additionally yields the following theorem.

TheOREM 2. For $n_{y} \leq n \leq n_{m+1}=m(T G+T Y+T R)$

$$
L \leq s_{k}^{n} \text { and } 0 \leq u_{k}^{n} \leq \mathcal{V}\left(s_{k}^{n}\right), \quad 1 \leq k \leq N-1,
$$

and

$$
0 \leq u_{N}^{n} \leq \mathcal{V}_{\infty}=\lim _{s \rightarrow \infty} \mathcal{V}(s)
$$

Moreover, for $1 \leq I \leq M$

$$
x_{p_{I}}^{n_{m+1}} \leq l_{I} .
$$

Theorems 1 and 2 go a long way towards establishing the consistency of our model. What remains to be shown is that cars with index $p_{I}+1$ clear the light, i.e., that they satisfy

$$
x_{\left(p_{I}+1\right)}^{n_{y}+\frac{T Y}{\Delta t}} \geq l_{I}+w+L .
$$

The reader should recall that the cars with these indices satisfy

$$
x_{\left(p_{I+1)}\right.}^{n_{y}}<l_{I} \text { and } x_{\left(p_{I}+1\right)}^{n_{y}}+\left(\begin{array}{c}
\min u_{j}^{n_{y}} \\
\left(p_{I}+1\right) \leq j \leq k_{I}
\end{array}\right) T Y \geq l_{I}+w+L
$$

and that cars with indices $\left(p_{I}+1\right) \leq k \leq k_{I}$ evolve by the standard discrete dynamics for $n_{y} \leq n \leq n_{y}+T Y / \Delta t-1$; i.e.,

$$
x_{k}^{n+1}=x_{k}^{n}+u_{k}^{n} \Delta t \text { and } u_{k}^{n}=\mathcal{V}\left(s_{k}^{n}\right)+\left(1-\frac{\Delta t}{\epsilon}\right)^{n-n_{y}}\left(u_{k}^{n_{y}}-\mathcal{V}\left(s^{n_{y}}\right)\right),
$$


where

$$
0 \leq u_{k}^{n_{y}} \leq \mathcal{V}\left(s_{k}^{n_{y}}\right) \text { and } L \leq s_{k}^{n} .
$$

It is a straightforward calculation to show that cars with these indices also satisfy

$$
\begin{aligned}
& u_{k}^{n+1}=\mathcal{V}\left(s_{k}^{n}+\left(u_{k+1}^{n}-u_{k}^{n}\right) \Delta t\right)+\left(1-\frac{\Delta t}{\epsilon}\right)\left(u_{k}^{n}-\mathcal{V}\left(s_{k}^{n}\right)\right) \\
& =\Delta t \mathcal{V}^{\prime}\left(s_{\#}\right) u_{k+1}^{n}+\left(1-\Delta t \mathcal{V}^{\prime}\left(s_{\#}\right)\right) u_{k}^{n}+\frac{\Delta t}{\epsilon}\left(\mathcal{V}\left(s_{k}^{n}\right)-u_{k}^{n}\right)
\end{aligned}
$$

from some $s_{\#} \geq L$, and that this identity, along with

$$
\Delta t \mathcal{V}^{\prime}(L) \leq 1, \quad \Delta t \leq \epsilon, \text { and } 0 \leq \mathcal{V}\left(s_{k}^{n}\right)-u_{k}^{n}
$$

implies

$$
u_{k}^{n+1} \geq \min \left(u_{k}^{n}, u_{k+1}^{n}\right) .
$$

We now note that at $t=t_{y}$ (equivalently $n=n_{y}$ ) the cars with indices $p_{I} \leq k$ typically satisfy

$$
\min _{p_{I} \leq j \leq k_{I}} u_{j}^{n_{y}}=u_{k_{0}}^{n_{y}}, \quad \text { where }\left(p_{I}+1\right) \leq k_{0} \leq k_{I},
$$

and

$$
u_{k+1}^{n_{y}}-u_{k}^{n_{y}} \geq 0, \quad k_{0} \leq k \leq k_{\#},
$$

where $k_{\#}$ is greater than $k_{I}$. Moreover, if the spacing of the lights is sufficiently large, then the spatial monotonicity of the velocities is preserved for $n_{y} \leq n \leq n_{y}+$ $T Y / \Delta t$ and $k_{0} \leq k \leq k_{\#}$. When this is the case, the inequalities (2.78)-(2.81) guarantee (2.77).

3. Simulations. In this section we present some simulations of the system outlined in section 1 . We chose

$$
\mathcal{V}_{\infty}=50 f / s, \quad L=20 f, \quad L_{1}=25 f, \quad \lambda=5, \quad \epsilon=5 s \text { and } N=600 .
$$

Our maximal velocity was given by

$$
\mathcal{V}(s)=\mathcal{V}_{\infty}\left(1-\frac{L}{s}\right), \quad L \leq s .
$$

We restrict our attention to a roadway with two in-phase lights located at

$$
l_{1}=1 \text { mile }=5280 f \quad \text { and } \quad l_{2}=2 \text { miles }=10,560 f,
$$

and we assume that the width of each intersection is

$$
w=20 f .
$$

Finally, the durations of the green, yellow, and red lights were chosen to be

$$
T G=25 s, \quad T Y=5 s, \quad \text { and } \quad T R=30 s .
$$



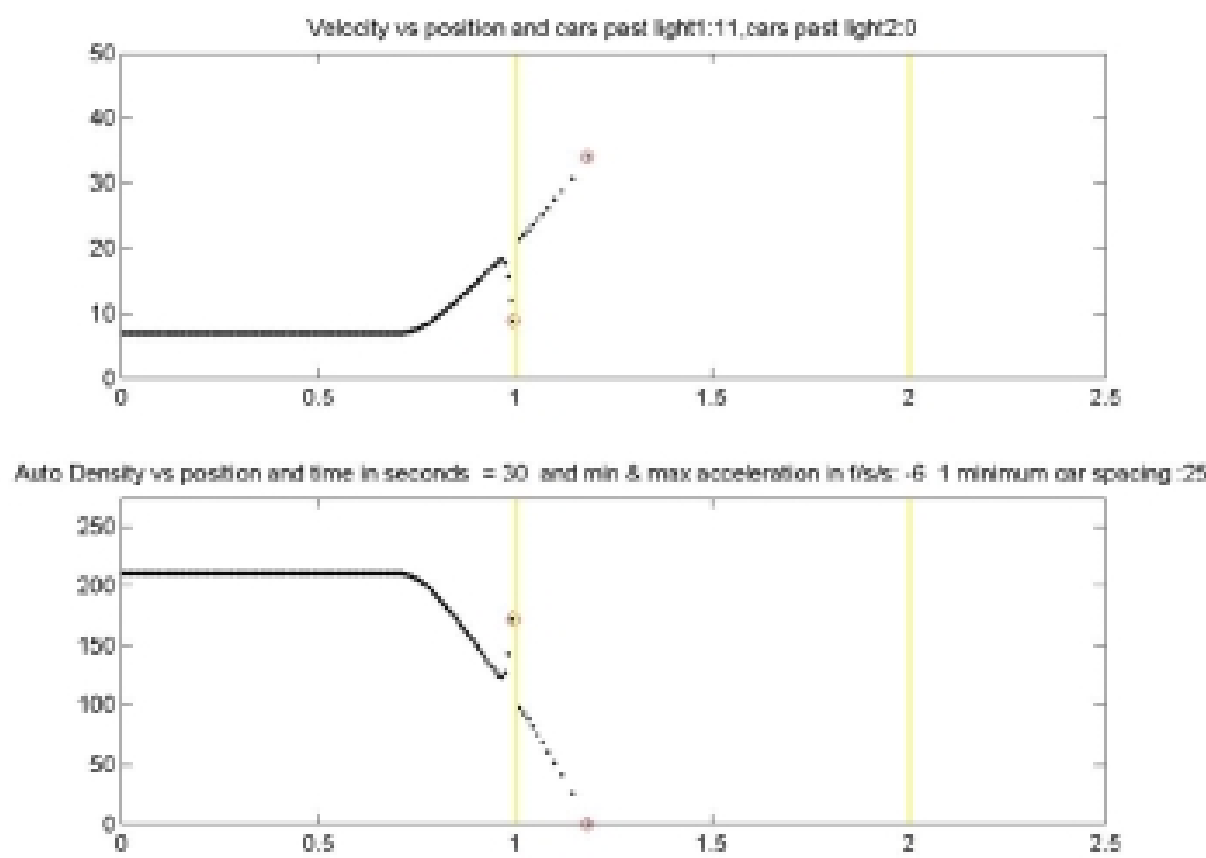

FIG. 1.

Our initial data are taken to be

$$
x_{k}(0)=25(k-400) \text { and } u_{k}(0)=0,1 \leq k \leq 600 .
$$

Snapshots of the solution are shown at times 30,147, 151, 179, and 191 seconds in Figures 1-5, respectively, and a film may be seen at http://www.math.cmu.edu/ users/plin/21380/traffic.html.

In the first frame of each snapshot we plot the auto velocity $u_{k}$ (in miles/hour) versus current auto position $x_{k}$ (in miles), and in the second frame we plot the empirical density $\rho_{k}=\frac{1}{x_{k+1}-x_{k}}$ (in cars/mile) versus current auto position $x_{k}$ (in miles).

After an initial startup period we are able to get 18 cars through each light during each green-yellow-red cycle. This number should be contrasted with what one obtains in the singular limit, where $\epsilon=0^{+}, T Y=0 s, T G=30 s, w=0 f$, and $\lambda=5$. In this limit

$$
u_{k} \equiv \mathcal{V}_{\infty}\left(1-\frac{L}{x_{k+1}-x_{k}}\right)
$$

and if, perchance, we have a car satisfying

$$
x_{k}\left(\left(t_{m}+T G\right)^{-}\right)=l_{I}, \quad I=1 \text { or } 2,
$$

and

$$
u_{k}\left(\left(t_{m}+T G\right)^{-}\right)>0
$$



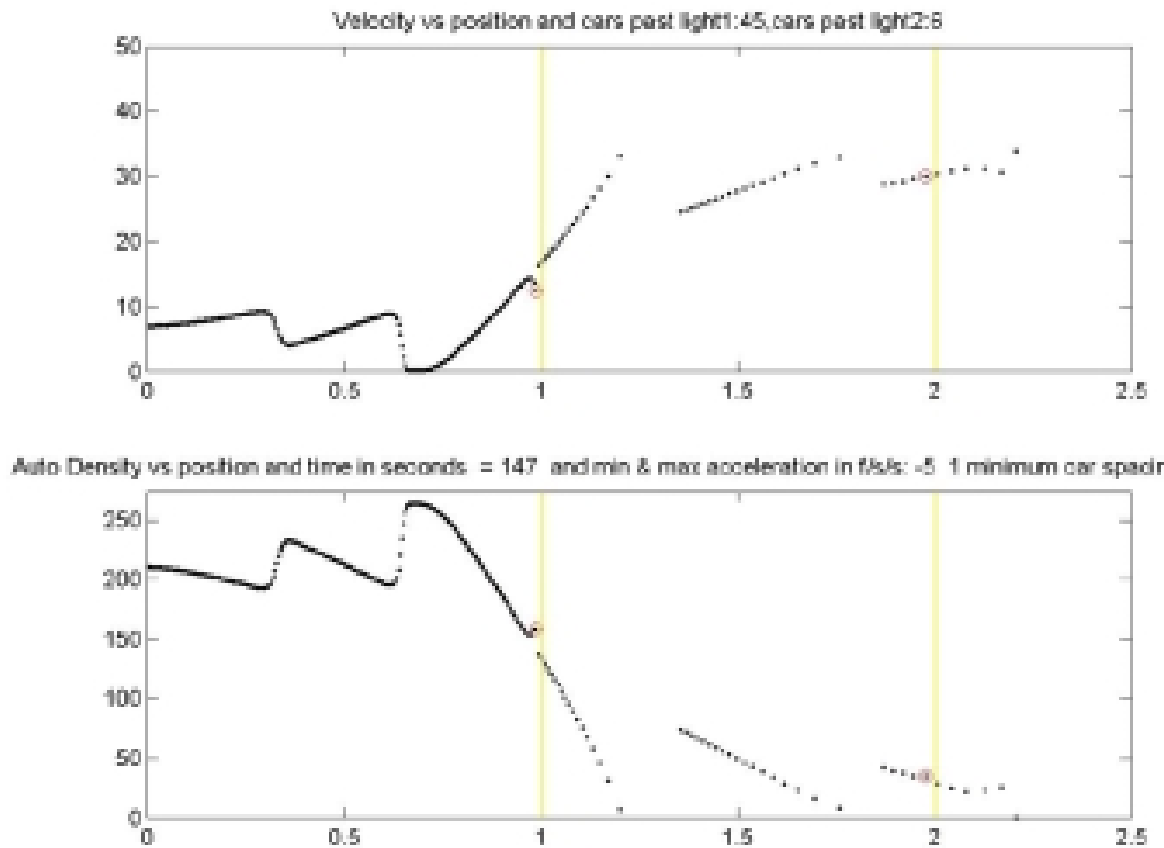

FIG. 2.

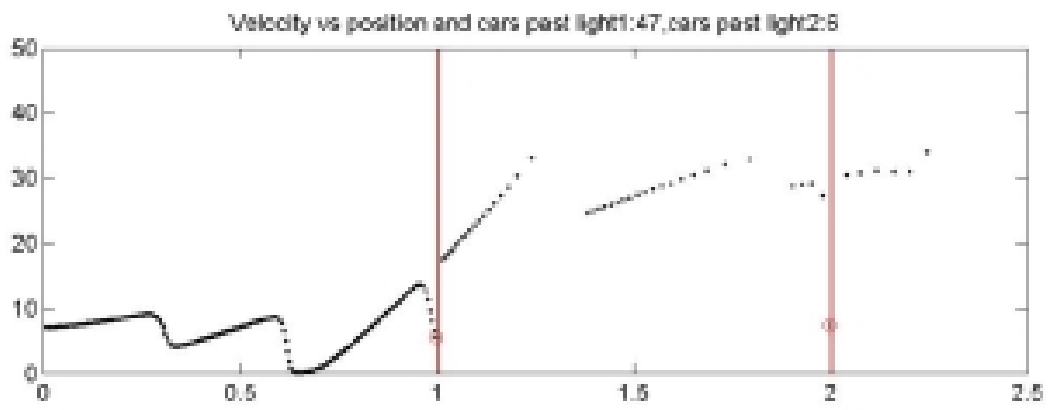

Auto Density vs position and tme in seconds $=151$ and min 8 max scceleration in fisis $-10 \quad 0$ minimum car spacing 20,03

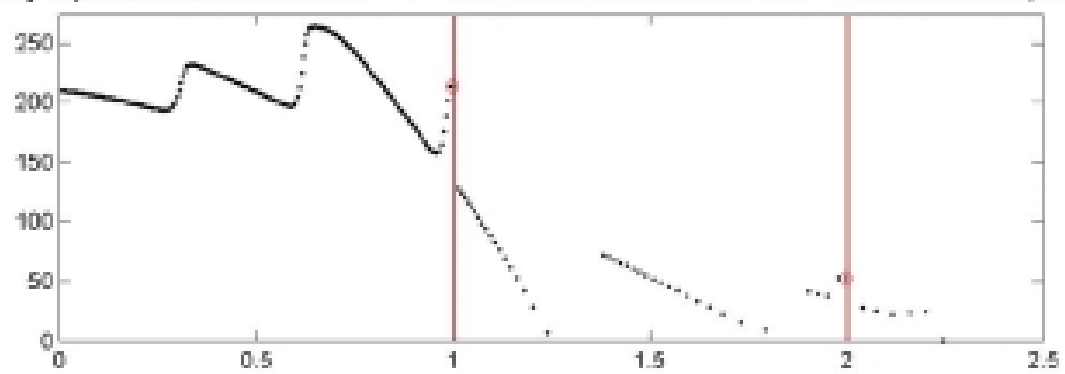

FIG. 3. 

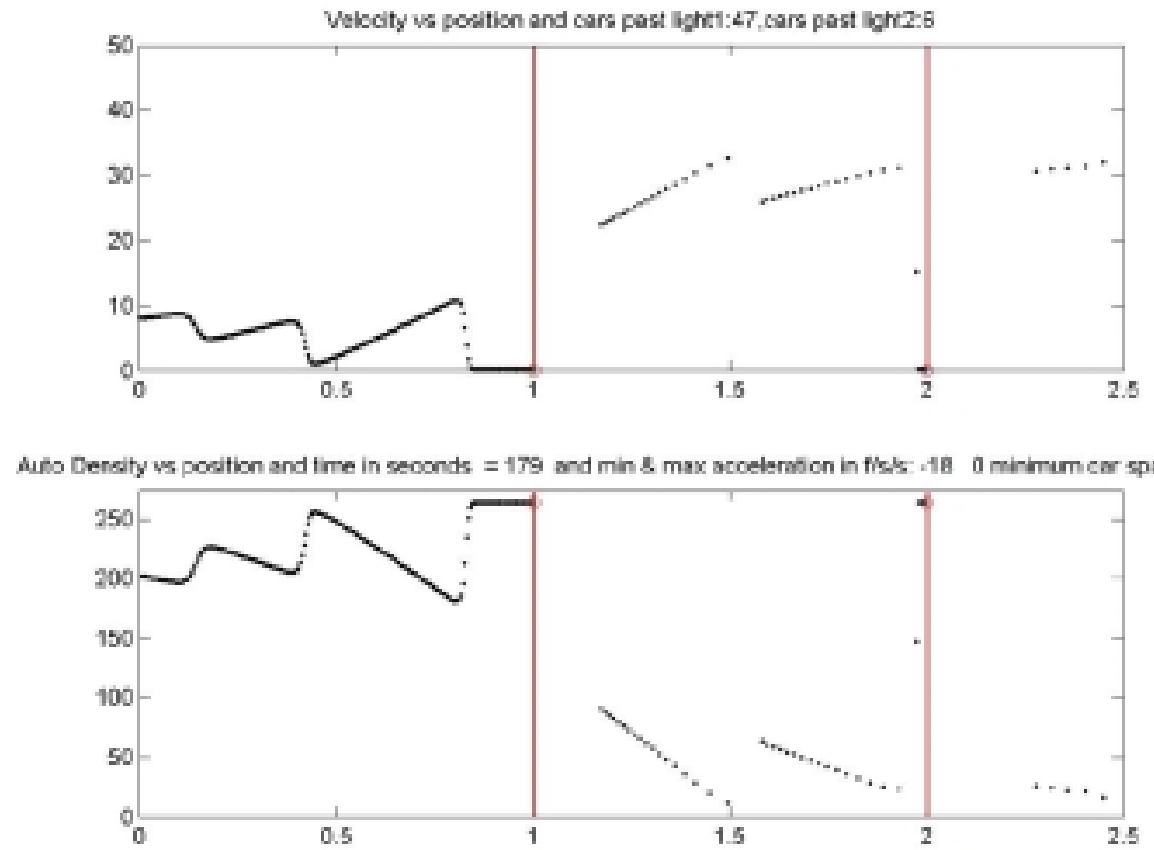

FIG. 4.

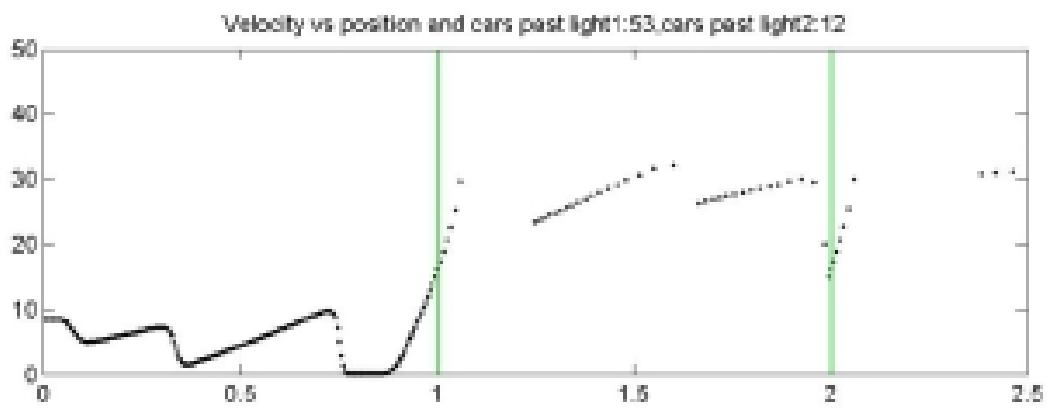

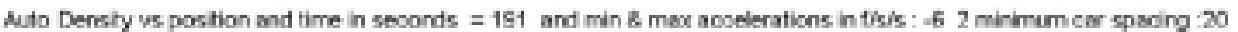

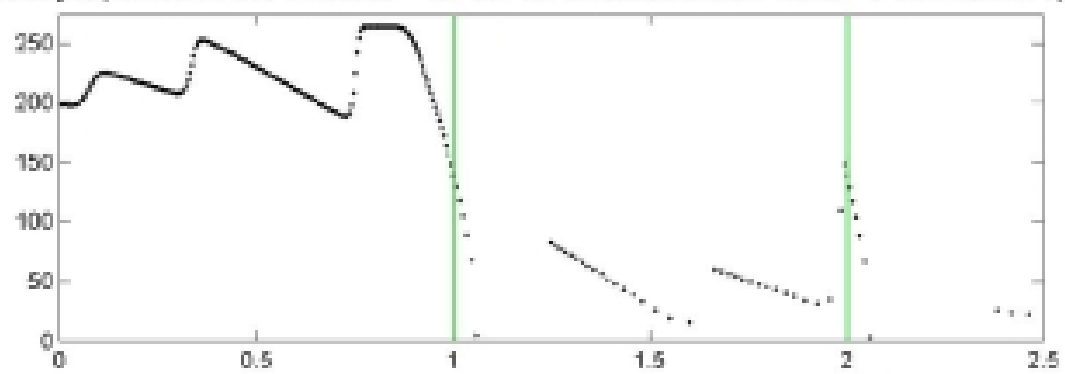

FIG. 5. 
then for times $t_{m}+T G<t \leq t_{m}+T G+T R$,

$$
x_{k}(t)=l \text { and } u_{k}(t) \equiv 0 \text {. }
$$

For this singular model we declare a car through the light at $l$ if $x_{k}\left(t_{y}\right)>l$. The singular model has the potential for infinite accelerations. In steady state the singular model allows us to get 20 cars through an intersection during each green-red cycle.

We note that our choice of which car must stop is made at times $t_{y}=t_{m}+T G$ (when a green light turns yellow) and is conservative when the car chosen to stop satisfies $x_{p_{l}}\left(t_{y}\right)<l-\lambda L$. A more aggressive strategy would have been to allow the $p_{l}$ th car to follow its standard dynamics until time $t_{p_{l}}<t_{y}+T Y$, where $x_{p_{l}}\left(t_{p_{l}}\right)=l-\lambda L$, and then reevaluate whether the $p_{l}$ th car can get through the light in the remaining time $t_{y}+T Y-t_{p_{l}}$, i.e., check whether

$$
x_{p_{l}}\left(t_{p_{l}}\right)+\min _{p_{l} \leq k \leq k_{l}\left(t_{p_{l}}\right)} u_{k}\left(t_{p_{l}}\right)\left(t_{y}+T Y-t_{p_{l}}\right) \geq l+w+L .
$$

If the latter inequality holds, the aggressive strategy would allow the $p_{l}$ th car through and stop the $\left(p_{l}-1\right)$ st car. We avoided this strategy because it did not seem to be worth the effort to get one more car through the intersection during the green-yellowred cycle.

The attentive reader will by now realize that once we have determined which car will slow down or stop at a given light the particular braking strategy adopted is immaterial; all that is required is that the velocity associated with the braking strategy, $v_{p_{l}}$, be such that if $x_{p_{l}}$ satisfies

$$
\frac{d x_{p_{l}}}{d t}=v_{p_{l}} \text { and } x_{p_{l}}\left(t_{y}\right)<l,
$$

then $x_{p_{l}}\left(t_{m+1}\right) \leq l$. We adopted constant braking strategies here because they were simple and realistic.

4. Concluding remarks. There are some obvious connections between the discrete model studied in this paper and the continuum or macroscopic models of Aw, Klar, Materne, and Rascle [3].

If one assumes that the maximal velocity $\mathcal{V}(\cdot)$ introduced in (1.1)-(1.3) is actually a function of $\gamma=\frac{s}{L}$ defined on $\gamma=\frac{s}{L} \geq 1$, i.e.,

$$
\mathcal{V}(s)=W\left(\frac{s}{L}\right)
$$

then (1.1) and (1.7) take the form

$$
\frac{d x_{k}}{d t}=u_{k} \text { and } \frac{d u_{k}}{d t}=W^{\prime}\left(\gamma_{k}\right)\left(\frac{u_{k+1}-u_{k}}{L}\right)+\frac{\left(W\left(\gamma_{k}\right)-u_{k}\right)}{\epsilon},
$$

where again

$$
\gamma_{k}=\frac{\left(x_{k+1}-x_{k}\right)}{L}
$$

and

$$
\frac{d \gamma_{k}}{d t}=\frac{u_{k+1}-u_{k}}{L}
$$


The connection between the follow-the-leader system (4.1)-(4.4) is now clear. One introduces reference coordinates

$$
X_{k}=k L,
$$

lets

$$
\mathcal{X}\left(X_{k}, t\right)=x_{k}(t) \text { and } u\left(X_{k}, t\right)=u_{k}(t),
$$

and interprets $\gamma_{k}$ and $\frac{u_{k+1}-u_{k}}{L}$ as the downwind finite difference approximations to $\frac{\partial \mathcal{X}}{\partial X}$ and $\frac{\partial u}{\partial X}$ at the reference point $X_{k}$; i.e.,

$$
\frac{\partial \mathcal{X}}{\partial X}\left(X_{k}, t\right)=\gamma_{k}=\frac{x_{k+1}-x_{k}}{L} \text { and } \frac{\partial U}{\partial X}\left(X_{k}, t\right)=\frac{u_{k+1}-u_{k}}{L} .
$$

With these identifications one obtains, at least formally, the Lagrangian traffic equations

$$
\frac{\partial \mathcal{X}}{\partial t}(X, t)=u(X, t) \text { and } \frac{\partial \mathcal{X}}{\partial X}=\gamma(X, t)
$$

where

$$
\frac{\partial \gamma}{\partial t}=\frac{\partial u}{\partial X} \text { and } \frac{\partial u}{\partial t}=W^{\prime}(\gamma) \frac{\partial u}{\partial X}+\frac{(W(\gamma)-u)}{\epsilon} .
$$

This correspondence is faithful if one restricts one's attention to initial value problems exclusively. We have not seen how to incorporate the traffic light problem into a continuum format.

\section{REFERENCES}

[1] J.M. Greenberg, Extensions and amplifications of a traffic model of Aw and Rascle, SIAM J. Appl. Math., 62 (2001), pp. 729-745.

[2] A. Aw AND M. RASCle, Resurrection of "second order" models of traffic flow, SIAM J. Appl. Math., 60 (2000), pp. 916-938.

[3] A. Aw, A. Klar, T. Materne, and M. Rascle, Derivation of continuum traffic flow models from microscopic follow-the-leader models, SIAM J. Appl. Math., to appear.

[4] M.J. Lighthill and G.B. Whitham, On kinematic waves. I: Flood movement in long rivers, Proc. Roy. Soc. London Ser. A, 229 (1955), pp. 281-316.

[5] M.J. Lighthill and G.B. Whitham, On kinematic waves. II: A theory of traffic flow on long crowded roads, Proc. Roy. Soc. London Ser. A, 229 (1955), pp. 317-345.

[6] P.I. Richards, Shock waves on the highway, Oper. Res., 4 (1956), pp. 42-51.

[7] G.B. Whitham, Linear and Nonlinear Waves, Pure and Applied Mathematics, WileyInterscience, New York, 1974.

[8] R. Rotherby, Car following models, in Traffic Flow Theory - A State of the Art Report, Special Report 165, Transportation Research Board, Washington, DC, 1975, Chapter 4; also available online from http://www.tfhrc.gov/its/tft/chap4.html.

[9] H.M. Zhang, A non-equilibrium traffic model devoid of gas-like behavior, Transportation Res. B, 36 (2002), pp. 275-290. 